\title{
The identification of pathogenic yeast strains by electrophoretic analysis of their chromosomes
}

\author{
M. MONOD, S. PORCHET, F. BAUDRAZ-ROSSELET and E. FRENK
}

Service de Dermatologie, Centre Hospitalier Universitaire Vaudois (CHUV-BH19), 1011 Lausanne. Switzerland

\begin{abstract}
Summary. Epidemiological studies require characterisation of pathogenic yeasts at and below the species level. The chromosomes of 130 strains of four pathogenic species of the genus Candida, isolated from clinical material, were separated by pulsed field electrophoresis with the clamped homogeneous electric field (CHEF) technique. Each species was characterised by a distinct electrophoretic karyotype (EK). Furthermore, smaller variations of the EK amongst strains belonging to the same species appeared to offer a useful means of strain differentiation. A karyotyping system is proposed for $C$. albicans. The EKs were assigned to a code of four numbers which designated the number of bands that could be resolved in each of four sets of chromosomes. Morphotypes of the colonies of $C$. albicans on malt agar plates, which did not correlate with the EK, could provide a complementary means of strain characterisation in epidemiological studies.
\end{abstract}

\section{Introduction}

Infections with opportunistic yeasts of the genus Candida are becoming more frequent. The species determination is achieved routinely by the results of sugar fermentation tests, although $C$. albicans, which is most frequently isolated from clinical specimens, can be identified selectively by germtube formation in human serum. ${ }^{1}$ An epidemiological approach requires characterisation below the species level. Previous studies reporting strain identification and differentiation, generally concerned with $C$. albicans, included morphotyping, ${ }^{2,3}$ biotyping by a series of plate tests, ${ }^{4,5}$ resistogram typing, ${ }^{6-8}$ serotyping, ${ }^{9}$ differential susceptibility to killer toxins, ${ }^{10}$ immunoblotting, ${ }^{11}$ and DNA fingerprinting. ${ }^{12}$ Recently, C. albicans was reported to have a variable karyotype by orthogonal field agarose gel electrophoretic (OFAGE) analysis performed with clinical isolates. ${ }^{13}$

Resolution of DNA fragments larger than $50 \mathrm{~kb}$ cannot be achieved by conventional agarose gel electrophoresis. Pulsed field gel electrophoresis (PFGE) was introduced for resolving DNA of chromosomal size in yeasts ${ }^{14,15}$ and protozoa. ${ }^{16}$ Contour clamped homogeneous electric field (CHEF) gel electrophoresis, the most recent devel-

Received 14 Sep. 1989; accepted 16 Nov. 1989. opment of the basic PFGE, allows good resolution, with preservation of sharp bands, straight lanes and reproducible separation of DNA molecules greater than $5000 \mathrm{~kb}^{17}$ Preliminary work has shown that this technique can be used in routine work for screening Candida strains. Therefore, the electrophoretic karyotype (EK) was investigated in 130 strains of four pathogenic species of the genus Candida, isolated from clinical material.

\section{Materials and methods}

\section{Yeast isolates}

The test strains -100 isolates of $C$. albicans and 10 each of C. parapsilosis, C. glabrata (formerly Torulopsis glabrata) and $C$. tropicalis-were isolated from 130 different patients at the Centre Hospitalier Universitaire Vaudois. All strains were grown on Sabouraud Agar and maintained on agar slants at $4^{\circ} \mathrm{C}$.

Isolates were identified as $C$. albicans by germ tube production in human serum. ${ }^{1}$ Strains that did not produce germ tubes were identified on the basis of sugar fermentation tests (API system).

\section{Sample preparation for electrophoresis}

Cells were grown overnight in Sabouraud Broth to late logarithmic phase at $30^{\circ} \mathrm{C}$. They were then harvested and washed with $50 \mathrm{~mm}$ EDTA, $\mathrm{pH} 8$, and $100 \mu$ l of the final 
cell pellet resuspended in $150 \mu \mathrm{l}$ of $50 \mathrm{~mm}$ EDTA, pH 8, were treated at $37^{\circ} \mathrm{C}$ for $20 \mathrm{~min}$ with $80 \mu$ of lyticase solution containing (/ml) $2 \mathrm{mg}$ of enzyme (Sigma 5263) in $10 \mathrm{~mm}$ sodium phosphate, $\mathrm{pH} 8$, and glycerol $50 \%$; $300 \mu \mathrm{l}$ of the cell-enzyme suspension were mixed with $1 \mathrm{ml}$ of low gelling-temperature agarose $1 \% \mathrm{w} / \mathrm{v}$ prepared in $125 \mathrm{~mm}$ EDTA, $\mathrm{pH} 7.5$ and cooled to $50^{\circ} \mathrm{C}$. The mixture was poured into mould chambers and allowed to cool to $4^{\circ} \mathrm{C}$ for $20 \mathrm{~min}$. The blocks of treated cells in gellified agarose were recovered and incubated overnight at $37^{\circ} \mathrm{C}$ in LETbuffer $(0.5 \mathrm{M}$ EDTA, pH 8.0, $0.01 \mathrm{M}$ Tris, $\mathrm{pH} \mathrm{7.5,2-mercaptoethanol} 7.5 \%)$. They were subsequently incubated for $24 \mathrm{~h}$ at $50^{\circ} \mathrm{C}$ with proteinase $\mathrm{K}$ (Boehringer 1.000 .144$), 1 \mathrm{mg} / \mathrm{ml}$ in NDS buffer $(0.5 \mathrm{M}$ EDTA, pH 8.0, 0.01 M Tris, pH 7.5, sodium laurylsarcocinate $1 \%$ ). Finally, the agarose plugs were washed three times for $24 \mathrm{~h}$ with $50 \mathrm{~mm}$ EDTA, pH 8.0, at room temperature, and stored at $4{ }^{\circ} \mathrm{C}$ in the same solution in a test tube.

\section{CHEF electrophoresis}

The chromosomes of each strain were separated by pulse field electrophoresis on agarose gels by the CHEF technique (contour clamped homogeneous electric field), in a CHEF-DR II system (Bio-Rad, USA). Pieces of $10 \times 2 \times 1 \mathrm{~mm}$ cut from sample preparation plugs were used to load the wells of the gels. The resolution of chromosomal DNA was performed in a $10 \mathrm{~cm} \times 10 \mathrm{~cm}$ agarose $0.9 \%$ gel, run at $150 \mathrm{~V}$ at a 120 -s switch for $24 \mathrm{~h}$, followed by a 180 -s switch for $16 \mathrm{~h}$.
Saccharomyces cerevisiae chromosomal DNA size standards, expressed in kilobases $(\mathrm{kb})$ were obtained from Biorad, USA.

Gels were stained with ethidium bromide $(0.5 \mu \mathrm{g} / \mathrm{ml})$ for $20 \mathrm{~min}$ and washed with distilled water for $5 \mathrm{~min}$. DNA bands were identified under UV light $(254 \mathrm{~nm})$. Photographs were taken with Polaroid type 667 film through a red filter (Kodak wratten 22) in a MP6 Polaroid camera.

\section{Morphotype}

Strains were streaked on malt extract agar (Oxoid) 6\% as described by Hunter $e t$ al., 1989 and Phongpaichit $e t$ al., 1987. ${ }^{2,3}$ After incubation for 10 days at room temperature, each strain was examined and assigned a morphotype code.

\section{Results}

The EK of the strains of Candida showed profiles which were characteristic for each of the four species (figs. 1, 2, 3). Variations of the band pattern were due to differences both in the number of bands and in their migration distance. Differences observed allowed differentiation not only at the species level, but also between strains belonging to the same species (figs. 1 and 2). The EK of $C$. tropicalis appeared to be less variable than that of the three other species, C. albicans, C. parapsilosis and $C$. glabrata.

\section{Code}

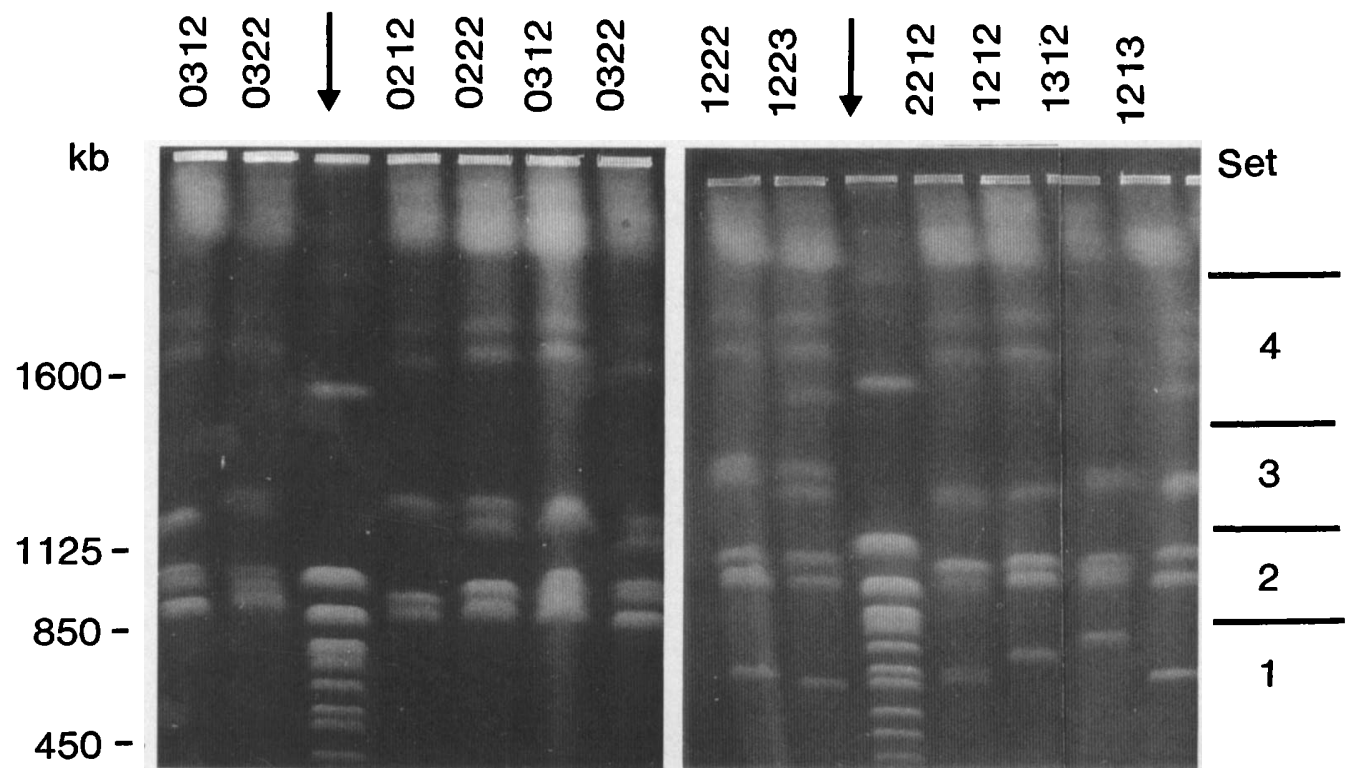

Fig. 1. Variation of the EK of 12 strains of $C$. albicans. Chromosomes of the yeast $S$. cerevisiae (arrow) were used as DNA size standards (indicated in $\mathrm{kb}$ on the left of the figure). The strains of $C$. albicans are assigned to a code of four numbers which designate the number of bands that can be resolved in four sets of chromosomes. The four sets are shown on the right of the figure. 

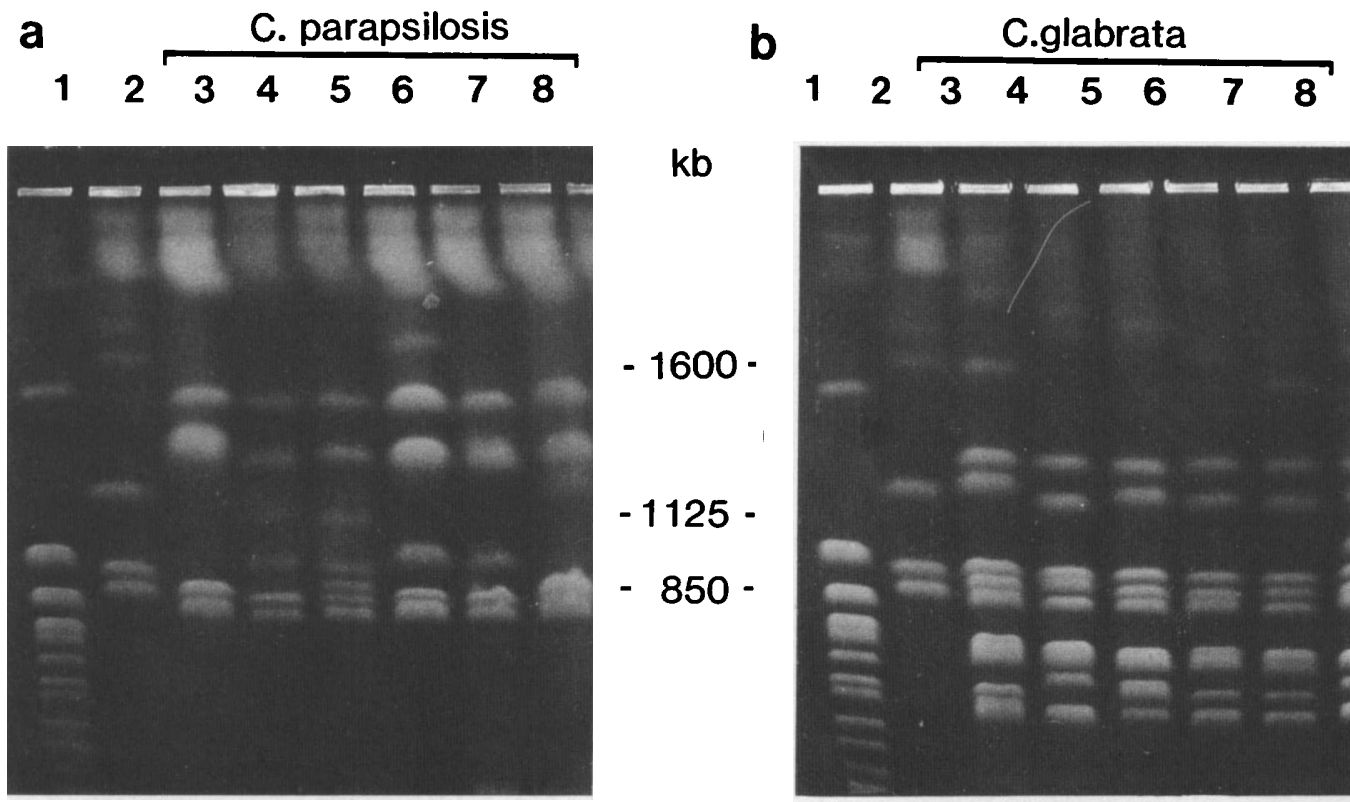

$\mathrm{kb}$

$-1600-$

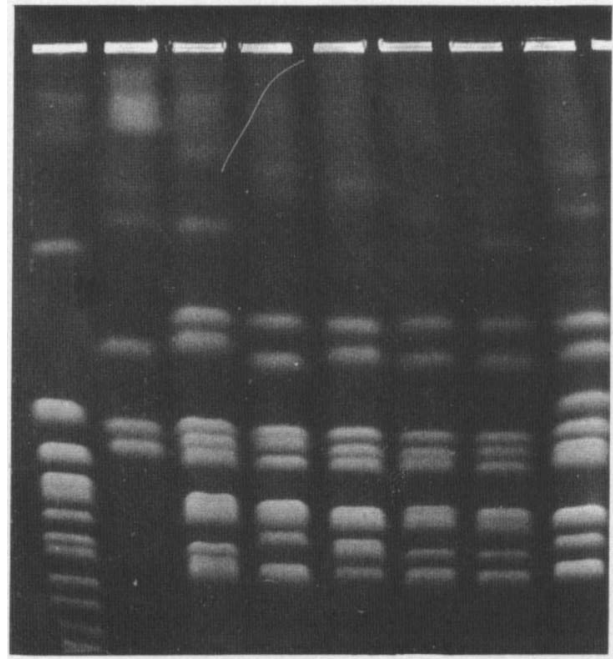

C

$-1125-$

$-850-$

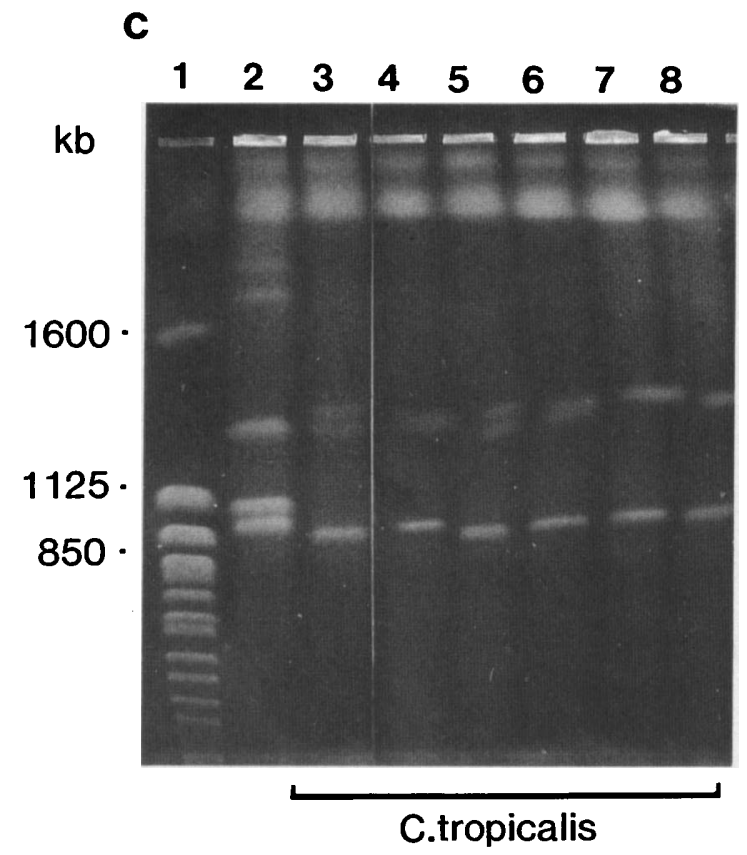

Fig. 2. Variation of the EK of 6 strains each of C.parapsilosis (lines A3-A8), C. glabrata (lines B3-B8), and C. tropicalis (lines C3C8). The chromosomes of $S$. cerevisiae (lines A1, B1, C1) are used as DNA size standards. The EK of six strains of the three yeast species is compared to the EK of a strain of C. albicans (no. 76 in our collection, EK code 0212) used as a reference (lines A2, B2, C2).

When the CHEF electrophoresis patterns of 100 clinical isolates of $C$. albicans were studied, bands which were single in some strains appeared to be double in others. Eleven bands have previously been resolved in C. albicans and four double bands turned out to be homologous chromosomes, ${ }^{18}$ which results in seven genetically independent chromosomes. In the numbering system of Magee et al. ${ }^{18}$ the largest chromosome was called no. 1 and the remainder were numbered successively in order of 


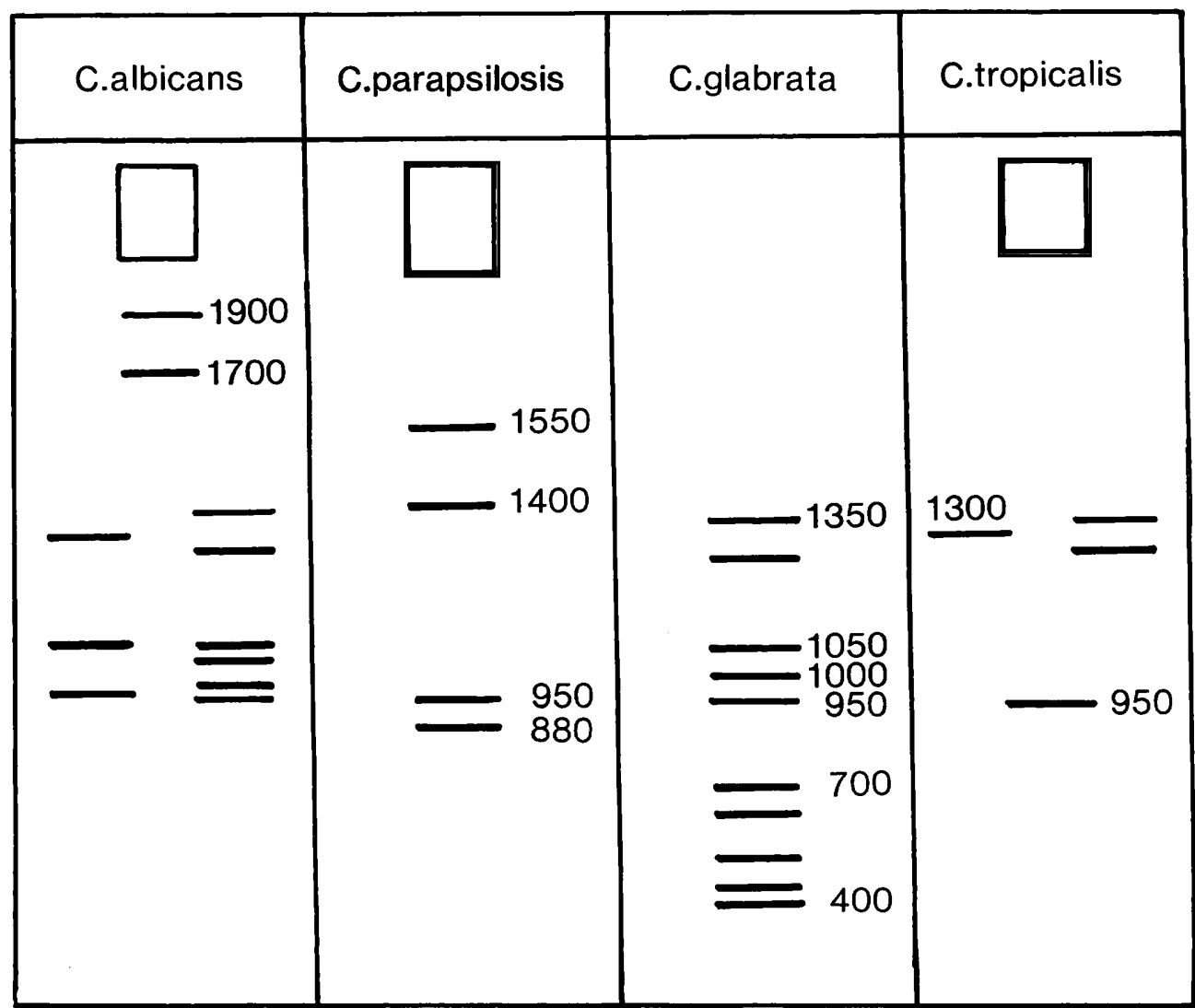

Fig. 3. Characteristic band patterns of $C$. albicans, C. parapsilosis, C. glabrata and C. tropicalis. For $C$. albicans and $C$. tropicalis, bands which were single in some strains appeared to be double in others. The size of the bands is indicated (in kb) when it was constant in $90-100 \%$ of the strains. The size of the other bands varied between the strains. The chromosomes larger than $2000 \mathrm{~kb}$ are represented by a square. Bands occasionally present are not drawn on this diagram.

increasing distance of migration. For practical purposes we assigned the EK of the strains of $C$. albicans to a code of four numbers (fig. 1). These numbers designate the number of bands that could be resolved in four sets of chromosomes under the electrophoresis conditions used. Variations between strains occurred mainly in the chromosomes with smaller sizes. Consequently, the number of bands in the set of chromosomes migrating furthest was written in the first position. The first number designates the bands of less than $850 \mathrm{~kb}$. DNA of this size was found in only eight strains which have a chromosome that apparently corresponded to none of the seven previously identified. ${ }^{18}$ This eighth chromosome appeared to be diploid as attested by a double band observed in the EK of one strain (fig. 1, class 2212). The second and third numbers refer to bands between 850 and $1125 \mathrm{~kb}$, Magee's chromosomes nos. 6 and 7, and between 1125 and $1600 \mathrm{~kb}$, Magee's chromosome no. 5, respectively. ${ }^{18}$ The latter three chromosomes are
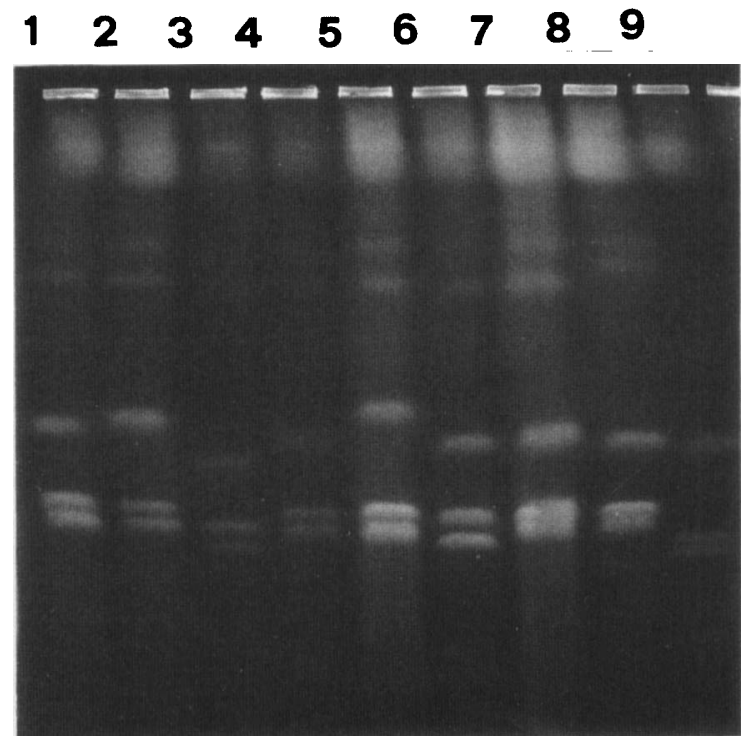

Fig. 4. Electrophoretic karyotypes of nine strains of C. albicans belonging to class 0212 . 
Table. Distribution of $100 \mathrm{C}$. albicans strains isolated from various sites

\begin{tabular}{|c|c|c|c|c|c|}
\hline \multirow[b]{2}{*}{ EK code } & \multicolumn{4}{|c|}{ Number of strains isolated from } & \multirow[b]{2}{*}{ Total } \\
\hline & $\begin{array}{c}\text { genito- } \\
\text { urinary } \\
\text { tract } \\
(24)\end{array}$ & $\begin{array}{c}\text { oral } \\
\text { cavity } \\
(32)\end{array}$ & $\begin{array}{l}\text { skin } \\
(26)\end{array}$ & $\begin{array}{l}\text { deep } \\
\text { infec- } \\
\text { tions } \\
(18)\end{array}$ & \\
\hline 0212 & 5 & 17 & 15 & 6 & 43 \\
\hline 0222 & 10 & 6 & 5 & 5 & 26 \\
\hline 0312 & 6 & 1 & 4 & 5 & 16 \\
\hline 0322 & 0 & 3 & 2 & 2 & 7 \\
\hline 1212 & 1 & 1 & $\ldots$ & $\ldots$ & 2 \\
\hline 1213 & $\ldots$ & 1 & $\ldots$ & $\ldots$ & 1 \\
\hline 1222 & 1 & 1 & $\cdots$ & $\cdots$ & 2 \\
\hline 1223 & $\cdots$ & 1 & $\cdots$ & $\ldots$ & 1 \\
\hline 1312 & $\cdots$ & 1 & $\ldots$ & $\cdots$ & 1 \\
\hline 2212 & 1 & $\cdots$ & $\cdots$ & $\ldots$ & 1 \\
\hline
\end{tabular}

diploid and can show pairs of homologous bands. The fourth number is generally 2 , and corresponds to the chromosomes nos. 3 and 4 which are haploid. ${ }^{18} \mathrm{~A}$ third band was observed in some strains. Chromosomes larger than $2000 \mathrm{~kb}$ were not considered in our analysis. Using this code the strains of $C$. albicans could be separated into 10 classes (table). Their corresponding EK pattern is shown in fig. 1. Classes $0212(43 \%)$ and $0222(26 \%)$ were the most frequently found. The EK of the strains in the same class were not identical, with variations occurring in the exact position of the bands in the gel (fig. 4). Co-migration in adjacent wells of the chromosomal DNA of two such strains, or co-migration with the chromosomal DNA of a strain chosen as standard, may be necessary to achieve further strain differentiation.

Many different morphotypes were observed when strains of $C$. albicans were streaked on malt extract

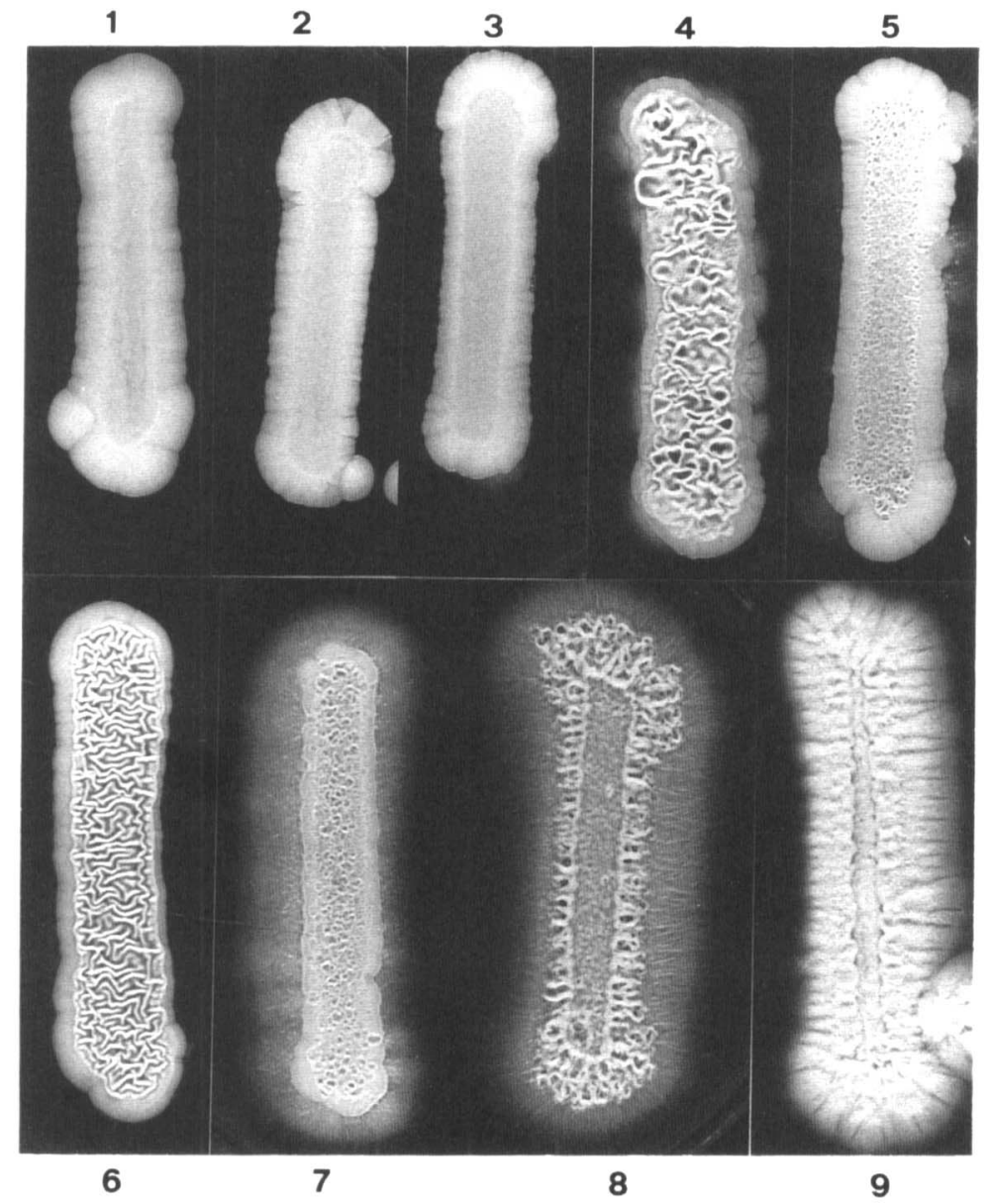

Fig. 5. Morphotype variation of nine strains of $C$. albicans with EK of the class 0212 . The EK of each strain, numbered 1-9, is shown in fig. 4. 
agar. $^{2,3}$ After incubation for 10 days at room temperature, yeasts with an EK belonging to the classes 0212,0222 and 0312 exhibited colonies with or without a fringe, and smooth, rough, cratered or wrinkled surface. Morphotype and EK appeared to be independent characters as illustrated by comparing figs. 4 and 5. The other classes, containing a small number of specimens, were not examined for correlations between morphotype and EK.

\section{Discussion}

Conditions of electrophoresis were chosen for the resolution of bands between 500 and $3000 \mathrm{~kb}$, which allows a separation of the chromosomes of Candida thought to be optimal for strain identification. Different chromosome preparations of the same strain gave identical band patterns under the same conditions of electrophoresis. Five transfers on conventional Sabouraud medium did not alter the EK of two strains each of $C$. albicans, $C$. parapsilosis, C. glabrata and C. tropicalis which were tested as a control. CHEF electrophoresis appears to be a simple and reproducible method of differentiation at and below the species level. The interpretation of the results is unequivocal and does not depend on the reader. Interestingly, in this study, strains of $C$. albicans with a small chromosome $(400-650 \mathrm{~kb})$ supplementary to the seven previously reported by Magee et al. ${ }^{18}$ were found.

\section{REFERENCES}

1. Taschdjian C L, Burchall J J, Kozinn P J. Rapid identification of Candida albicans by filamentation on serum and serum substitutes. Am J Dis Child 1960; 99: 212-215.

2. Phongpaichit S, Mackenzie D W R, Fraser C. Strain differentiation of Candida albicans by morphotyping. Epidemiol Infect 1987; 99 : 421-428.

3. Hunter P R, Fraser C A M, Mackenzie D W R. Morphotype markers of virulence in human candidal infections. $J$ Med Microbiol 1989; 28 : 85-91.

4. Odds, F C, Abbott A B. A simple system for the presumptive identification of Candida albicans and differentiation of strains within the species. Sabouradia 1980;18: 301317.

5. Odds F C, Abbott A B. Modification and extension of tests for differentiation of Candida species and strains. Sabouraudia $1983 ; 21$ : 79-81.

6. Warnock D W, Speller D C E, Day J K, Farrell A J. Resistogram method for differentiation of strains of Candida albicans. J Appl Bacteriol 1979; 46: 571-578.

7. McCreight M C, Warnock D W. Enhanced differentiation of isolates of Candida albicans using a modified resistogram method. Mykosen 1982; 25: 589-598.
These strains were identified as $C$. albicans by germ tube production in human serum, and the identity was confirmed by sugar fermentation.

Strains of $C$. albicans were also differentiated on the basis of morphology of colonies streaked on malt agar. ${ }^{2,3}$ Morphotyping appeared to be independent of the EK. These methods appear to be complementary and, used in conjunction, could yield a finer strain characterisation in epidemiological studies.

No clear association between a given EK and the anatomical source of the isolates was found (table) although a striking finding was the low and high proportion of EK of classes 0212 and 0222 respectively in isolates from the genitourinary tract. Further studies are needed to confirm or invalidate this unexpected result for which no obvious explanation is evident.

Interestingly there are data that indicate that strains showing identical EK can be isolated from different body sites in the same patient. ${ }^{13}$ Currently, the origin of the yeasts involved in systemic candidiasis occurring in immunocompromised patients, and in chronic mucocutaneous infections, is being investigated, and electrophoresis with the CHEF technique should permit a more precise approach.

We thank Dr J. Bille for providing some $C$. tropicalis and $C$. glabrata strains, $\mathrm{Dr} \mathrm{H}$. Pooley for critical reading of the manuscript, Dr D. Pittet for helpful discussions and J. Chevalley for secretarial assistance.
8. McCreight M C, Warnock D W, Martin M V. Resistogram typing of Candida albicans isolates from oral and cutaneous sites in irradiated patients. Sabouraudia 1985; 23: 403-406.

9. Hasenclever H F, Mitchell W O. Antigenic studies of Candida. 1. Observation of two antigenic groups in Candida albicans. J Bacteriol 1961 ; 82: 570-573.

10. Polonelli L, Archibusacci C, Sestito M, Morace G. Killer system: a simple method for differentiating Candida albicans strains. J Clin Microbiol 1983; 17: 774-780.

11. Lee W, Burnie J, Matthews R. Fingerprinting Candida albicans. J Immunol Methods 1986; 93: 177-182.

12. Scherer S, Stevens D A. Application of DNA typing methods to epidemiology and taxonomy of Candida species. $J$ Clin Microbiol 1987; 25: 675-679.

13. Merz W G, Connelly C, Hieter P. Variation of electrophoretic karyotypes among clinical isolates of Candida albicans. J Clin Microbiol 1988; 26: 842-845.

14. Carle G F, Olson M V. Separation of chromosomal DNA molecules from yeast by orthogonal-field-alternation gel electrophoresis. Nucleic Acids Res 1984; 12: 56475664.

15. Schwartz D C, Cantor C R. Separation of yeast chromosomesized DNAs by pulsed field gradient gel electrophoresis. Cell $1984 ; 37: 67-75$. 
16. Van der Ploeg L H T, Schwartz D C, Cantor C R, Borst P. Antigenic variation in Trypanosoma brucei analyzed by electrophoretic separation of chromosome-sized DNA molecules. Cell 1984; 37 : 77-84.

17. Vollrath D, Davis R W. Resolution of DNA molecules greater than 5 megabases by contour-clamped homo- geneous electric fields. Nucleic Acids Res 1987; 15: $7865-7876$.

18. Magee B B, Koltin Y, Gorman J A, Magee P T. Assignment of cloned genes to the seven electrophoretically separated Candida albicans chromosomes. Mol Cell Biol $1988 ; 8: 4721-4726$. 